\title{
Investigating iron deficiency anemia without clinical evidence of gastrointestinal blood loss
}

\author{
Subrata Ghosh MBBS MD FRCPC FRCP FRCPE, Editor-in-Chief
}

I: ron deficiency anemia is a common indication for referral to gastroenterologists. The referral triage process includes complete blood count including mean corpuscular volume, iron status by serum ferritin, iron and transferrin saturation and celiac serology. An often posed question is whether to investigate patients with iron deficiency without anemia - these patients, if older than 50 years of age, are at a higher risk of gastrointestinal malignancy compared with subjects with normal iron status and, therefore, should be investigated for gastrointestinal blood loss (1). All patients should undergo a detailed medical history and examination to exclude dietary insufficiency and menorrhagia. An ultrasound scan of the abdomen and pelvis may be useful to rule out a gynecological cause or a renal carcinoma.

Patients with iron deficiency anemia and no overt or occult evidence of gastrointestinal bleeding are generally investigated by upper and lower gastrointestinal endoscopy. Patients with a hemoglobin concentration $<100 \mathrm{~g} / \mathrm{L}$ should be investigated urgently. It is reasonable to perform both upper endoscopy and colonoscopy - an effective strategy in those older than 50 years of age of age might be to perform colonoscopy with ileoscopy first and, if negative, perform upper endoscopy or limited push enteroscopy. Symptoms are a poor guide to the diagnostic yield of either procedure. Small intestinal biopsies should be taken irrespective of celiac serology.

Patients with iron deficiency anemia with no overt or occult evidence of gastrointestinal bleeding and negative upper endoscopy and colonoscopy present a diagnostic challenge. The available options include proceeding to small intestinal investigations, iron supplementation and proceeding to small intestinal investigations if anemia persists despite adequate iron supplementation, discontinuation of any potentially incriminating drugs, such as nonsteroidal anti-inflammatory drugs, despite negative investigations or simply wait and see with hemoglobin monitoring if the anemia is mild or hemoglobin is normal with iron deficiency. Wireless capsule endoscopy is commonly used in these patients as a first-line small intestinal investigation.

The study published in the current issue of the Canadian Journal of Gastroenterology by Tong et al (2) (pages 687-690), demonstrated that patients investigated with negative endoscopies for iron deficiency anemia without evidence of bleeding were generally managed with supportive therapy irrespective of positive or negative findings at wireless capsule endoscopy. Many of the positive findings at wireless capsule endoscopy were within the reach of conventional endoscopies.

Therefore, while obscure upper gastrointestinal bleeding is the most common indication for referral for wireless capsule endoscopy, a meaningful clinical decision change as a result of the findings is uncommon. Therefore, better selection criteria for referral to wireless capsule endoscopy are required (3). Otherwise, the impact of wireless capsule endoscopy in patients with iron deficiency anemia with no overt or occult bleeding and negative upper and lower gastrointestinal tract endoscopy is likely to be very limited.

The extent to which these challenging patients are investigated depends on comorbidities, the severity of anemia, whether the anemia is symptomatic, whether alternative routes of blood loss have been excluded and the rapidity with which anemia recurs after iron supplementation (4). The role of cross-sectional imaging, such as computed tomography (CT) or magnetic resonance-enterography, CT-angiography or double-balloon enteroscopy, in this specific patient group is uncertain. These investigations are generally performed in patients with definite clinical evidence of gastrointestinal blood loss.

A practical approach in these patients may be to institute supportive treatment with iron supplementation after negative upper and lower gastrointestinal tract endoscopies. If hemoglobin levels are restored and maintained on long-term iron supplementation, a watch-and-wait policy may be best. If hemoglobin levels cannot be normalized or drop while on iron supplementation, repeat upper and lower endoscopies may be performed before wireless capsule endoscopy. Double-balloon enteroscopy may be performed for confirming significant abnormalities detected at wireless capsule enteroscopy and to perform therapeutic interventions such as for vascular lesions (Figure 1). Small intestinal tumours or Crohn disease may require CT or magnetic resonance-enterography.

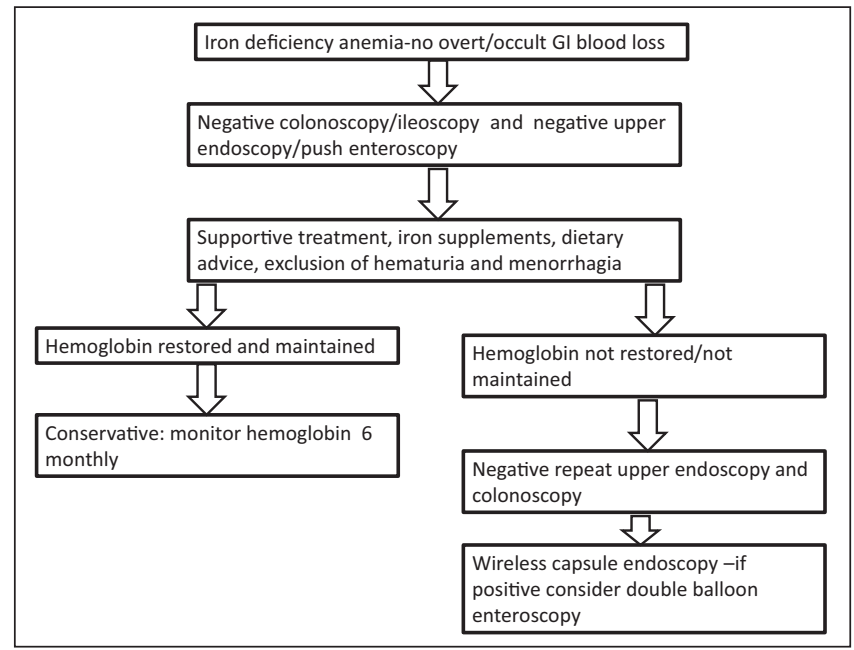

Figure 1) A pragmatic algorithm for investigating iron deficiency anemia with no evidence of overt or occult gastrointestinal bleeding

\section{REFERENCES}

1. Ioannou GN, Rockey DC, Bryson CL, Weiss NS. Iron deficiency and gastrointestinal malignancy: A population based cohort study. Am J Med 2002;113:276-80.

2. Tong J, Svarta S, Ou G, Kwok R, Law J, Enns R. Diagnostic yield of capsule endoscopy in the setting of iron deficiency anemia without evidence of gastrointestinal bleeding. Can J Gastroenterol 2012;26:687-90.

3. Liao Z, Gao R, Xu C, Li ZS. Indications and detection, completion and retention rates of small bowel capsule endoscopy: A systematic review. Gastrointest Endosc 2010;71:280-6.

4. Li X, Dai J, Lu H, Gao Y, Chen H, Ge Z. A prospective study on evaluating the diagnostic yield of video capsule endoscopy followed by directed double-balloon enteroscopy in patients with obscure gastrointestinal bleeding. Dig Dis Sci 2010;55:1704-10.

Department of Medicine, University of Calgary, Calgary, Alberta

Correspondence: Dr Subrata Ghosh, Division of Gastroenterology, Department of Medicine, University of Calgary, 3280 Hospital Drive

Northwest, Calgary, Alberta T2N 4N1. Telephone 403-944-8222, fax 403-944-1095, e-mail subrata.ghosh@albertahealthservices.ca Received and accepted for publication September 7, 2012 


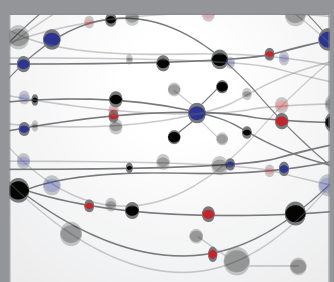

The Scientific World Journal
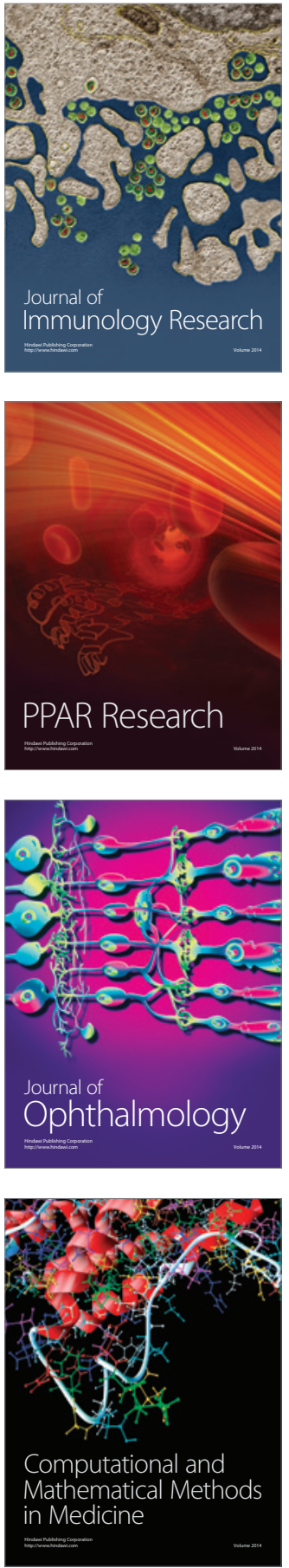

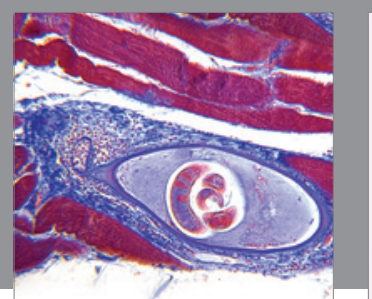

Gastroenterology Research and Practice



\section{Hindawi}

Submit your manuscripts at

http://www.hindawi.com
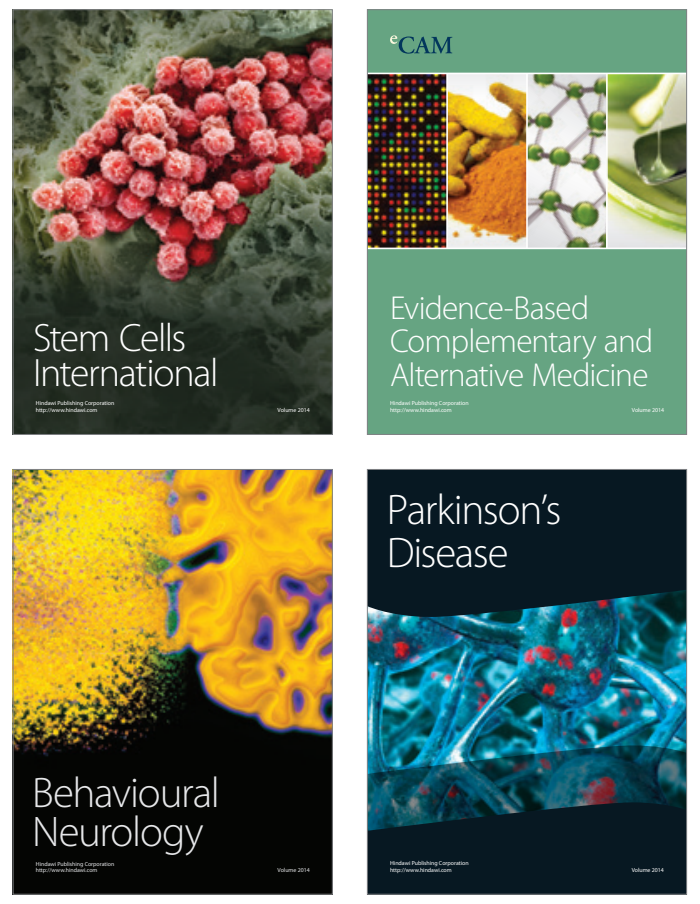
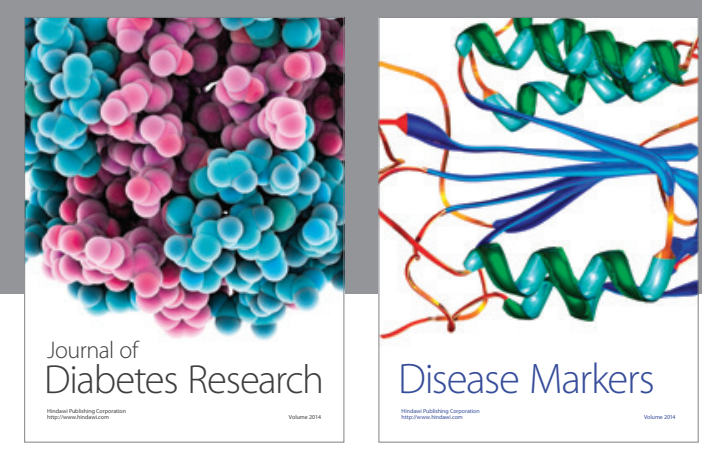

Disease Markers
